\title{
Genome-Wide Comparative Analysis of BZR1 Transcription Factor in Zea mays
}

\begin{abstract}
Naureen $Z^{1}$, Maqsood H², Mazhar MW', Mehmood J ${ }^{1}$ and Safeer Mehdi Bukhari SM${ }^{3}$

${ }^{1}$ Department of Bioinformatics and Biotechnology, Government College University Faisalabad, Pakistan ${ }^{2}$ Department of Plant Biotechnology, Asab, Nust, Pakistan ${ }^{3}$ Department of Biotechnology, University of Lahore, Pakistan

*Corresponding author: Muhammad Waqar Mazhar, Department of Bioinformatics and Biotechnology, Government College University Faisalabad, Pakistan
\end{abstract}

Received: J uly 06, 2021; Accepted: J uly 27, 2021; Published: August 03, 2021

\begin{abstract}
The BR-responsive genes are then regulated by BRASSINAZOLERESISTANT (BZR) Transcription Factors (TFs). As BRs possess numerous stress-resistant functions, BZR TFs also show activities of stress-resistance along with other developmental functions. Up to $88 \%$ similarity of protein sequences has been observed between BZR1 and BZR2 genes. Many positive roles of BZR TFs have been revealed by many studies in positively regulating the BR signal transduction in rice and maize family, but there is a very limited research is available on the BZR gene family of Zea mays. The aim of this study is to perform a wide-genome analysis of BZR1 transcription factor in Zea mays so that regulatory role of BZR TFs in BR-induced signaling pathway can be revealed. Gene structure analysis revealed the information about exons and introns. Phylogenetic analysis was done to identify the maximum likelihood among different families of BZR genes. Restriction analysis provided the information about the presence of restriction sites in Zea mays genome.
\end{abstract}

\section{Introduction}

During the plant lifecycle, developmental processes of plants need certain plant-specific hormones, called Brassinosteroids (BRs) [1]. Recently, different types of mutants have been used to study the BR signal transduction [2]. During the BR signal transduction, BRs bind to a memberane-localized receptor, BRASSINOSTEROIDINSENSITIVE 1 (BRI 1) [3]. The BR-responsive genes are then regulated by BRASSINAZOLE-RESISTANT (BZR) transcription factors (TFs) [4]. As BRs possess numerous stress-resistant functions, BZR TFs also show activities of stress- resistance along with other developmental functions [5]. As transcriptional activators and repressors, BZR1 and BZR2/BES1 are the gene members of BZR TF family along with homologs (BEH1-BEH4) [6]. For example, in Arabidopsis thaliana, transcription is suppressed by the in vivo identification of CGTG (T/C)G sequence by BZR1, which attaches to DWF4 and CPD promoters [7]. The promoters of BR-induced genes contain E box sequences (CANNTG) that bind to the BES1, which in turn recognizes BIM1-a helix-loop-helix protein [6,8].

Up to $88 \%$ similarity of protein sequences has been observed between BZR1 and BZR2 genes. Both BR-induced gene SAUR$\mathrm{AC} 1$ and BR-repressed DWF4 genes bind to BZR1 and BZR2 genes, according to chromatic immunosuppression quantitative PCR analysis $[9,10]$. The PCR analysis also shows that out of 19 BZR1 binding sites, BZR2 attaches to 18 of them [11]. Studies have also indicated that BZR TFs regulate other TFs to participate in plant development and growth [12]. For that reason, new insights into the BZR gene family can be obtained by identifying new BZR genes among different species of plants using reliable approaches.

In agricultural production, there are two main goals: improving crop quality and increasing crop yield. BRs are one of the critically important steroid hormones of plants responsible for a variety of cellular responses, such as pathogenic resistance, tolerance to environmental stresses, and cell elongation [13]. All these cellular responses help increase yield of crops. Many positive roles of BZR TFs have been revealed by many studies in positively regulating the $\mathrm{BR}$ signal transduction in rice and maize family, but there is a very limited research is available on the BZR gene family of Zea mays.

We analyzed BZR gene family of Zea mays by performing a comparative genome analysis of ZmBZR genes. First of all, published transcriptome analyses were used to identify ZmBZR gene. In this study, we focused on identification of BZR TFs in Zea mays., phylogenetic analysis, motif analysis, and gene structure analysis to predict the function of ZmBZR genes in Zea mays. The obtained results can be a new start to finding regulatory roles of BZR TFs in BR signaling pathway.

\section{Materials and Methods}

\section{Identification of BZR transcription factors}

The genomic and protein sequences of BZR1 transcription factor in Zea mays were retrieved from NCBI (https://www.ncbi.nlm.nih. gov/). The amino acid sequence of BZR1 from Arabidopsis was used as query sequence against non-redundant protein sequences databases of Zea mays. FASTA format of BZR1 protein sequence was used to run BLAST-P to find similar sequences. Pfam database (http://pfam.xfam.org/) was used to identify conserved domains and motifs of the candidate protein sequence. The conserved domains of BZR1 were further confirmed by multiple sequence alignment. Based on sequence alignment, MEGA 7.2 was used to align and create the maximum likelihood evolutionary tree of BZR1 proteins of Zea mays and A. thaliana.

\section{Identification of domains and motifs}

The sequence of BZR1 gene was used as query sequence and other five candidate sequences were downloaded to analyze the conserved domains and motifs. Pfam database ((http://pfam.xfam.org/) was used to identify conserved domain and motifs. All the domains were conserved except CL0061 and CL0167. All of the variants were 
Significant Dtam-A Matches

Shive or thide al aligmmeris

\begin{tabular}{|c|c|c|c|c|c|c|c|c|c|c|c|c|}
\hline \multirow{2}{*}{ Family } & \multirow{2}{*}{ Description } & \multirow{2}{*}{$\begin{array}{l}\text { Entry } \\
\text { type: }\end{array}$} & \multirow{2}{*}{ Clan } & \multicolumn{2}{|c|}{ Favelope } & \multicolumn{2}{|c|}{ Alignment - } & \multicolumn{2}{|c|}{ BMAM } & \multirow{2}{*}{ Henge } & \multirow{2}{*}{$\begin{array}{l}\text { Bit } \\
\text { sccire }\end{array}$} & \multirow{2}{*}{ Evalue } \\
\hline & & & & Stant & End & Start & End & From & To & & & \\
\hline Efesit B & 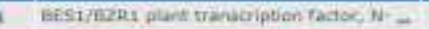 & Damain & 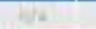 & 14 & 154 & is & 159 & 2 & 151 & 152 & 206.11 & $2 . \tan +11$ \\
\hline 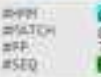 & 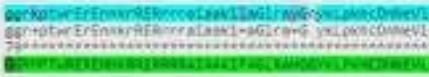 & 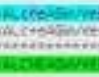 & 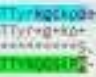 & $\sin 2$ & 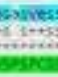 & Kess: & copesingas & 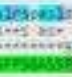 & 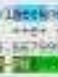 & 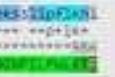 & & \\
\hline Bes: 14 & BESt/BZZU plant transolption factor. $\mathrm{N}$ - - & Dequant & $6 / 2$ & 359 & +90 & 360 & 420 & 2 & 151 & 152 & 206.8 & $2.10-61$ \\
\hline $\min _{\substack{x \rightarrow 0 \\
\text { side }}}$ & 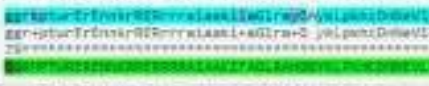 & 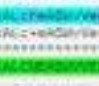 & 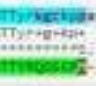 & Exint: & 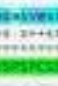 & $1 \mathrm{Th}$ & mexpes & ands: & inger & 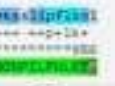 & & \\
\hline Exat $\mathrm{Ni}$ & 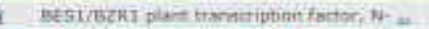 & Damain & ieto & 703 & $\$ 45$ & $7 n e$ & Bet & 2 & $15 x$ & 152 & 200.8 & $2,1+-51$ \\
\hline $\begin{array}{l}\text { net } \\
\text { atses }\end{array}$ & 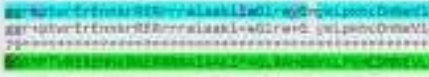 & 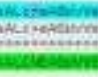 & 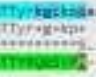 & 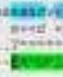 & 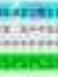 & 25 & 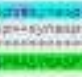 & 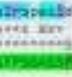 & anter & 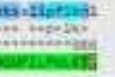 & & \\
\hline Besting & 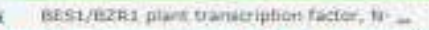 & Daman : & was & 1055 & 1305 & 1035 & $120=$ & 1 & 151 & $15 t$ & $202: 5$ & $\$ 4.40 \cdot 60$ \\
\hline 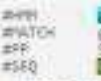 & 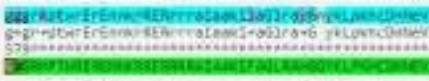 & 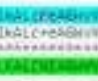 & 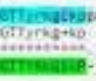 & gestid: & WT:35: & tess & 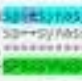 & 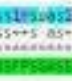 & wase & 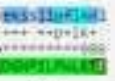 & & \\
\hline BESLI: & HESL/EZUS plare transoriphon factar, N- - & Domain & $\omega$ & 1999 & 1830 & 1390 & 153 h & 1 & 151 & 152 & 201.5 & =.6e-6p \\
\hline $\lim _{x \rightarrow 0}$ & 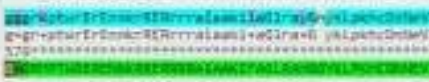 & Alarame & 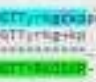 & intes: & & anes & 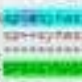 & 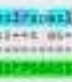 & ing & 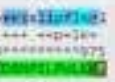 & & \\
\hline
\end{tabular}

Figure 1: Significant Pham-A matches.

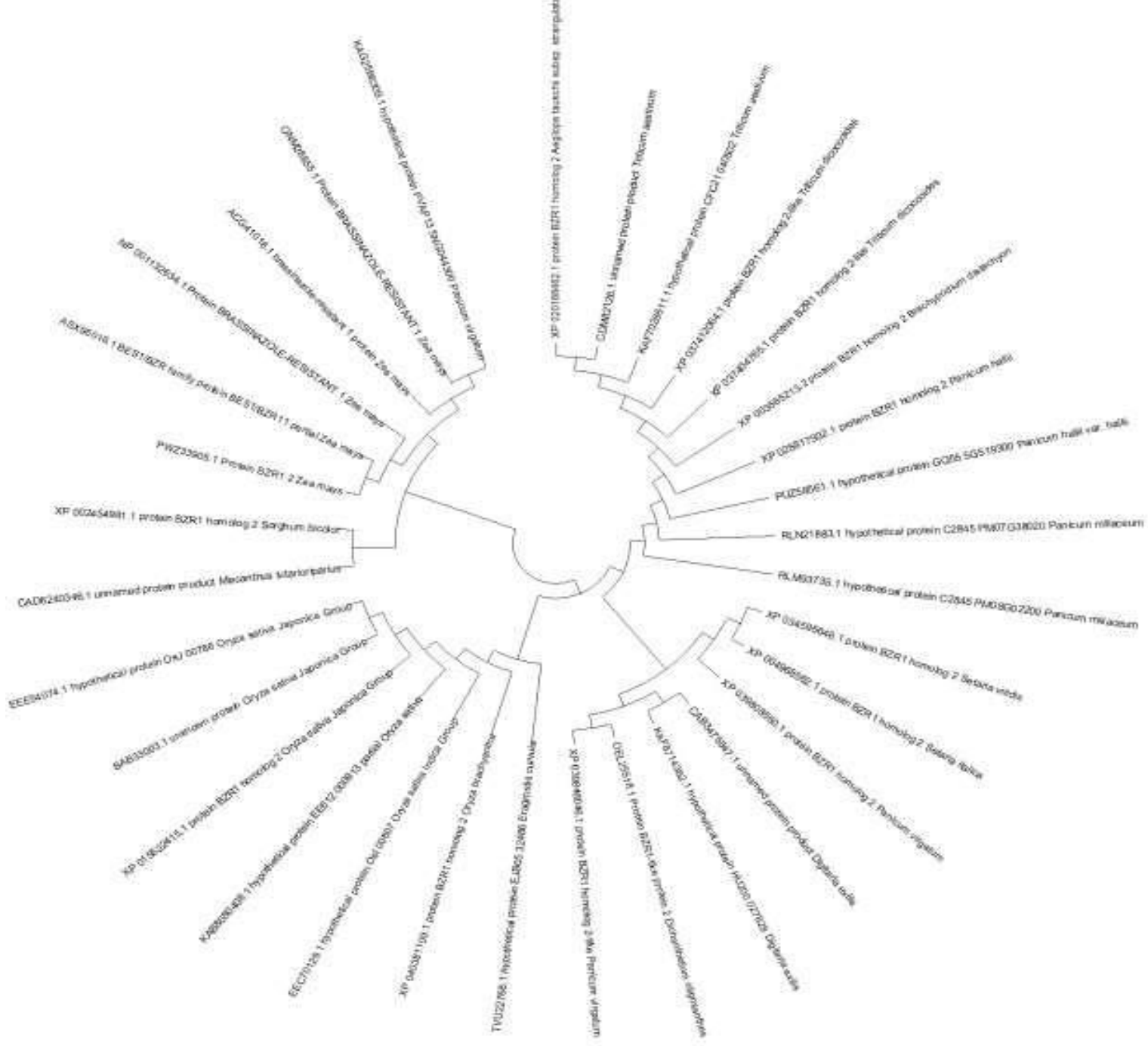

Figure 2: Phylogenetic Tree of BZR Transcription Family. 
carefully examined with E- value less than 1e-10 and those with lengthy ORF were chosen for further data analysis using various databases, (http://www.ncbi.nlm.nih.gov/Structure/cdd/wrpsb.cgi), (http://smart.embl- heidelberg.de/), and (http://pfam.janelia.org/). Position of chromosome, length of protein and cDNA and genomic sequences were observed by using NCBI database.

\section{Phylogenetic analysis}

The evolutionary relationship between the BZR genes of Zea mays, A. thaliana, Miscanthus lutarioriparius, and Oryza sativa was performed by aligning their sequences and using MEGA 7.2 to create rooted tress by maximum likelihood method. Tree topology and branch lengths are from a maximum likelihood analysis, with midpoint rooting. Nodes with single numbers indicate the results of a bootstrap maximum likelihood analysis with 12 replicates. Even after that, the Dlx gene family was classified based on their evolutionary similarity. A separate instrument was used to measure the molecular weight and isoelectric point toolbox (http://www. expasy.org/tools). Evolutionary analysis was used to name the subfamilies of the BZR gene based on their homologs. The evolutionary relationship among the families was performed using the online Time Tree Server.

\section{Gene structure analysis of BZR genes}

Both the coding sequences (mRNA and genomic sequences) and protein sequences were used to load positional information into Gene Structure Display Server. Gene Structure Display Server is an online database to assess information about introns/exons.

\section{Restriction analysis}

Restriction anlaysis of BZR gene was performed by loading the protein sequence of ZmBZR gene in Serial Cloner. Serial Cloner is software for mapping the sequence of proteins and identifying restriction sites contained in a graphic map. This tool also has the function of performing virtual PCR.

\section{Results}

\section{Identification of BZR1 gene in Zea mays}

The protein sequences of Zea mays and A. thaliana were retrieved from NCBI and used to run NCBI-BLAST. Identification of most possible matches was made more precise by using each query in separate BLAST searches. Redundant sequences were removed after all the queries. We identified that there are 11 BZR genes in Zea mays family. Pfam database was used to confirm the presence of conserved domains (Figure 1).

\section{Phylogenetic analysis by using MEGA 7.2}

A phylogenetic tree, also called phylogeny, is a diagram that shows how various plants, organisms, or genes derived from a

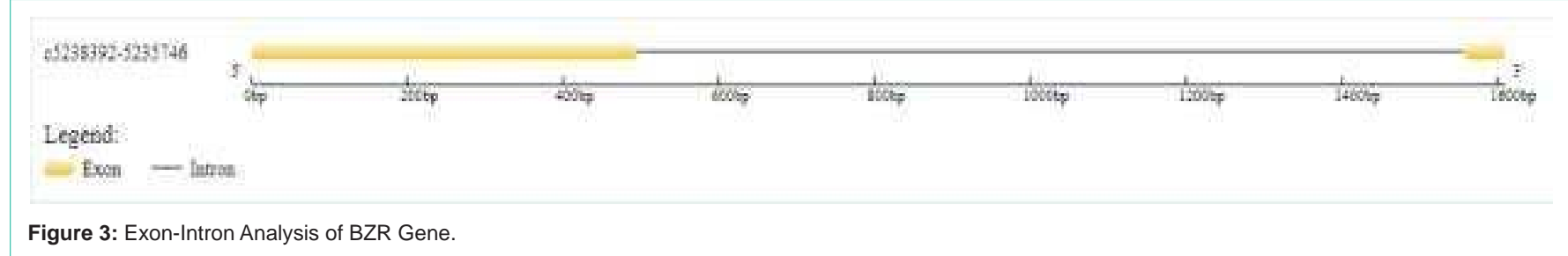

Figure 3: Exon-Intron Analysis of BZR Gene.

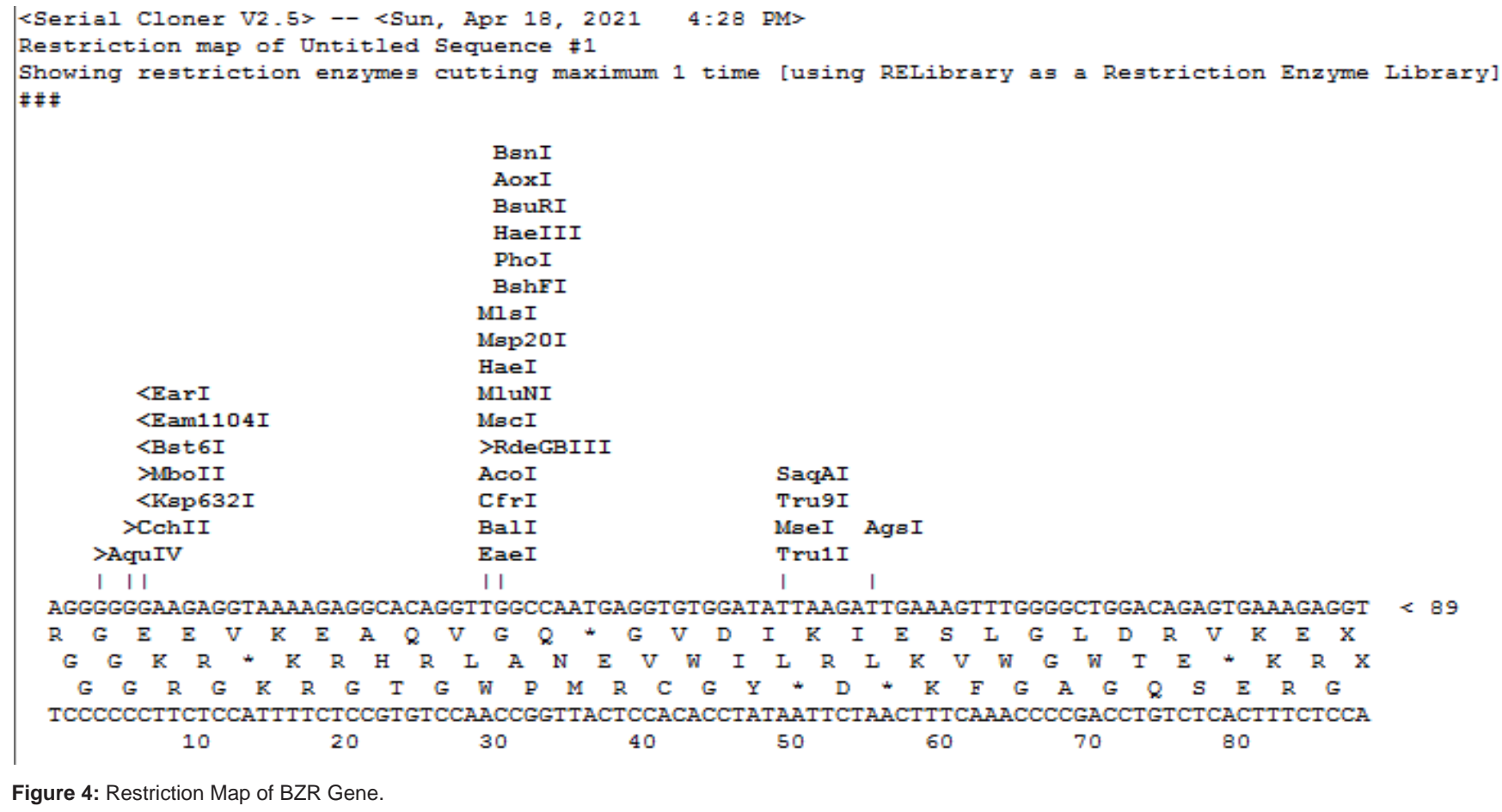

Figure 4: Restriction Map of BZR Gene. 
common ancestor. Phylogenies are useful for organising biological diversity records, structuring classifications, and gaining insight into evolutionary events. The rooted tree obtained from the BZRcontaining species was as follows (Supplementary Table S1 \& Figure 2).

\section{Gene structure analysis of BZR}

The visualization of BZR gene features such as the composition and location of exons, introns, and conserved domains were done using the Gene Structure Display Server (GSDS). The structural diversity of BZR genes was investigated by comparing CDS and genomic sequence of BZR genes in Zea mays to analyze introns and exons. A considerable diversity was observed in the length of exons (Figure 3).

\section{Restriction analysis of BZR gene}

The genomic and sequence map of BZR gene after restriction analysis using Serial Cloner showed different restriction sites, showing restriction enzymes cutting maximum 1 time (Figure 4).

\section{Discussion}

Identification of the role of BRs in responding to biotic and abiotic stress and regulating various physiological functions is the result of decades of research [14]. BR signal transduction and perception activates the role of BZR1 transcription factors, which not only activate thousands of genes, but also repress genes involved in BR biosynthesis [15]. As BRs cannot travel to long distances, they are transported by BZR1 transcription factors. One of the major functions of BZR1 is responding to biotic and abiotic stress and regulating gene expression [16]. Arabidopsis thaliana is the model dicot species for obtaining information about BRs [17]. BZR1 factors have also been studied in rice, but there's a limited validation to fully study the BZR1 TFs in maize.

In this work, a genome-wide comparative analysis was done to identify the role of BZR transcription factors in Zea mays. From the maize genome, 11 BZR genes were identified. However, Brassica and Arabidopsis contained 15 and 6 genes, respectively. Scanning of domains and motifs showed that conserved BZR motifs are present in all maize BZR. Study of functional domains has revealed the presence of highly conserved N-terminal domains in BZR transcription factors, which possess DNA binding affinity. We also performed a phylogenetic analysis of BZR transcription factors, which has revealed their maximum likelihood with other dicots.

To be concluding, it was confirmed that like BZR TFs present in Arabidopsis, BZR TFs regulate physiological role by exhibiting stress signaling activity in Zea mays.

\section{References}

1. Clouse SD. Molecular genetic studies confirm the role of brassinosteroids in plant growth and development. The Plant Journal. 1996; 10: 1-8.
2. Luo X-M, Lin, W-H, Zhu S, Zhu J-Y, Sun Y, Fan X-Y, et al. Integration of Lightand Brassinosteroid-Signaling Pathways by a GATA Transcription Factor in Arabidopsis. Developmental Cell. 2010; 19: 872-883.

3. Friedrichsen DM, Joazeiro CAP, Li J, Hunter T, Chory J. BrassinosteroidInsensitive-1 Is a Ubiquitously Expressed Leucine-Rich Repeat Receptor Serine/Threonine Kinase1. Plant Physiology. 2000; 123: 1247-1256.

4. Kim T-W, Guan S, Sun Y, Deng Z, Tang W, Shang J-X, et al. Brassinosteroid signal transduction from cell-surface receptor kinases to nuclear transcription factors. Nature Cell Biology. 2009; 11: 1254-1260.

5. Singh AP, Fridman $Y$, Friedlander-Shani L, Tarkowska D, Strnad M Savaldi-Goldstein S. Activity of the Brassinosteroid Transcription Factors BRASSINAZOLE RESISTANT1 and BRASSINOSTEROID INSENSITIVE1ETHYL METHANESULFONATE SUPPRESSOR1/BRASSINAZOLE RESISTANT2 Blocks Developmental Reprogramming in Response to Low Phosphate Availability. Plant Physiology. 2014; 166: 678-688.

6. Yin Y, Vafeados D, Tao Y, Yoshida S, Asami T, Chory J. A New Class of Transcription Factors Mediates Brassinosteroid-Regulated Gene Expression in Arabidopsis. Cell. 2015; 120: 249-259.

7. He J-X, Gendron JM, Sun Y, Gampala SSL, Gendron N, Sun CQ, et al BZR1 Is a Transcriptional Repressor with Dual Roles in Brassinosteroid Homeostasis and Growth Responses. Science. 2005; 307: 1634-1638.

8. Wang W, Sun YQ, Li GL, Zhang SY. Genome-wide identification, characterization, and expression patterns of the BZR transcription factor family in sugar beet (Beta vulgaris L.). BMC Plant Biology. 2019; 19: 1-12.

9. Ryu $\mathrm{H}, \mathrm{Kim} \mathrm{K}, \mathrm{Cho} \mathrm{H}$, Hwang I. Predominant actions of cytosolic BSU1 and nuclear BIN2 regulate subcellular localization of BES1 in brassinosteroid signaling. Molecules and Cells. 2010; 29: 291-296.

10. Yin Y, Wang Z-Y, Mora-Garcia S, Li J, Yoshida S, Asami T, et al. BES1 Accumulates in the Nucleus in Response to Brassinosteroids to Regulate Gene Expression and Promote Stem Elongation. Cell. 2002; 109: 181-191.

11. Ryu H, Kim K, Cho H, Park J, Choe S, Hwang I. Nucleocytoplasmic Shuttling of BZR1 Mediated by Phosphorylation Is Essential in \& It;em\&gt; Arabidopsis \& It;/em\&gt; Brassinosteroid Signaling. The Plant Cell. 2007; 19: 2749-2762.

12. Yu X, Li L, Zola J, Aluru M, Ye H, Foudree A, et al. A brassinosteroid transcriptional network revealed by genome-wide identification of BESI target genes in Arabidopsis thaliana. The Plant Journal. 2011; 65: 634-646.

13. Oh E, Zhu J-Y, Bai M-Y, Arenhart RA, Sun Y, Wang Z-Y. Cell elongation is regulated through a central circuit of interacting transcription factors in the Arabidopsis hypocotyl. ELife. 2014; 3: e03031.

14. Nawaz F, Naeem M, Zulfiqar B, Akram A, Ashraf MY, Raheel M, et al. Understanding brassinosteroid- regulated mechanisms to improve stress tolerance in plants: a critical review. Environmental Science and Pollution Research. 2017; 24: 15959-15975.

15. Zhu J-Y, Sae-Seaw J, Wang Z-Y. Brassinosteroid signalling. Development 2013; 140: 1615-1620.

16. Kir G, Ye H, Nelissen H, Neelakandan AK, Kusnandar AS, Luo A, et al. RNA Interference Knockdown of BRASSINOSTEROID INSENSITIVE1 in Maize Reveals Novel Functions for Brassinosteroid Signaling in Controlling Plant Architecture. Plant Physiology. 2015; 169: 826-839.

17. Saini S, Sharma I, Pati PK. Versatile roles of brassinosteroid in plants in the context of its homoeostasis, signaling and crosstalks. In Frontiers in Plant Science. 2015; 6: 950. 